\title{
Not all proteinuria is created equal
}

\author{
Andrew Beenken, Jonathan M. Barasch, and Ali G. Gharavi
}

Division of Nephrology, Department of Medicine, Columbia University Vagelos College of Physicians and Surgeons, New York, New York, USA.

\begin{abstract}
Albuminuria acts as a marker of progressive chronic kidney disease and as an indicator for initiation of hypertension treatment via modulation of the renin-angiotensin-aldosterone system with angiotensin receptor blockers or angiotensin-converting enzyme inhibitors. However, the true significance of albuminuria has yet to be fully defined. Is it merely a marker of underlying pathophysiology, or does it play a causal role in the progression of kidney disease? The answer remains under debate. In this issue of the $J C l$, Bedin et al. used next-generation sequencing data to identify patients with chronic proteinuria who had biallelic variants in the cubilin gene (CUBN). Through investigation of these pathogenic mutations in CUBN, the authors have further illuminated the clinical implications of albuminuria.
\end{abstract}

recent low-resolution electron microscopy data suggest that these C-terminal domains should cluster at the distal end of a 700-Å-long tree-like structure (7) where they are readily exposed to the urinary filtrate and available to bind proteins. In addition to albumin, it is possible that these domains could bind other ligands of similar size and isoelectric point ( $\mathrm{pI}$ ) such as vitamin D-binding protein (DBP), which has a close evolutionary relationship to albumin.

Varying proteinuric phenotypes have been reported in the literature, depending on which domains of cubilin are affected. Previously, a p.P1297L point mutation within the VitB12-IF binding site was observed to lead to anemia without proteinuria. Additionally, numerous variants causing global cubilin dysfunction have been documented to cause IGS with both anemia and proteinuria that is inclusive of albumin as well as other cubilin ligands such as apoA-1, transferrin, and DBP (8). Now, the study by Bedin et al. extends the known proteinuric phenotypes resulting from CUBN variants to include isolated albumin-predominant proteinuria resulting from C-terminal mutations (4). Certainly, further analysis of urinary proteins from patients with CUBN variants is bound to delineate still more phenotypes of proteinuria.

The authors noted two interesting features of the patients in the studied cohorts: (a) despite experiencing chronic proteinuria, these patients retained normal estimated glomerular filtration rates (eGFRs), a standard assessment of renal function, which is consistent with prior studies reporting normal renal function in patients with IGS, and (b) the proteinuria they exhibited was albumin predominant, with, on average, more than $60 \%$ of the total proteinuria consisting of albumin (4).

\section{The impact of albuminuria on renal function}

Albuminuria is a predictor of negative clinical outcomes including the progression of renal disease, but the degree to which albuminuria can be used independently as a surrogate therapeutic target is a matter of 
ongoing discussion (9-12). Furthermore, whether albuminuria causes progressive chronic kidney disease (CKD) also remains unknown. Causality has been suggested by reductions in albuminuria associated with reduced $\mathrm{CKD}$ progression to end-stage renal disease (ESRD) in several different clinical trials that evaluated the treatment of CKD patients with angiotensin-converting enzyme inhibitors (ACEIs) or angiotensin receptor blockers (ARBs) (13-16). Notably, the Renoprotection of Optimal Antiproteinuric Doses (ROAD) trial showed a reduction in CKD progression in nondiabetic proteinuric patients when renin-angiotensin-aldosterone system (RAAS) blockade was titrated to albuminuria targets (17), but the significance of those results has been disputed (10). So far, a reduction in albuminuria beyond that achieved by ACEI/ARB monotherapy has yet to show an effect on hard outcomes (18-22), raising the possibility that RAAS inhibition itself, rather than the associated reductions in albuminuria, mediates the clinical benefits.

There are reasonable grounds to posit a role for albuminuria in the pathogenesis of proteinuric CKD. In vitro evidence has shown that protein overload in proximal tubule cells activates $\mathrm{NF}-\kappa \mathrm{B}$ signaling, upregulates inflammation via monocyte chemotactic protein 1 , and induces vasoconstriction via endothelin 1 (23), which together can contribute to the development of tubulointerstitial disease. The in vivo evidence, however, does not fully support the hypotheses generated from in vitro experiments. Humans with loss of function of megalin or cubilin have up to $2.5 \mathrm{~g} / \mathrm{d}$ of proteinuria (1), implying that the proximal tubule is chronically exposed to gram quantities of albumin that must be efficiently endocytosed by megalin-cubilin. This suggests at least that chronic albumin exposure in the proximal tubule is insufficient for the development of tubulointerstitial disease.

The proteinuric consequences of cubilin mutations have been well documented (8), but Bedin et al. (4) went further to query the impact of albuminuria on renal function. Their 39 patients were primarily children, but the age range encompassed newborns, teenagers, seven patients aged 30 to 40 years, and one aged 66 years, all of whom exhibited normal eGFRs of great- er than $80 \mathrm{~mL} / \mathrm{min}$. Although the authors could not document an extended longitudinal follow-up of renal function measurements in their patients, their observation of normal or near-normal eGFRs in patients with decades of exposure to albuminuria is nonetheless intriguing. To further investigate their findings, the authors conducted a meta-analysis of cohorts from the CKDGen Consortium using CUBN variants previously associated with albuminuria in GWAS studies (24). Through this cross-sectional population-based approach and using an additive model to test for associations, the authors found an association of four different CUBN variants with both albuminuria and a small but statistically significant increase in eGFR. Thus, in these patients with CUBN loss of function and isolated chronic proteinuria, the consequence of long-term albumin exposure to the kidney tubules may be benign. The authors reanalyzed their data for one of the variants under a recessive model and found a stronger association with albuminuria in homozygotes. It will be informative to reanalyze all these GWAS data under a recessive model to confirm an association with more severe proteinuria in individuals carrying two of these risk variants. In addition, the availability of large data sets such as those from the UK Biobank will enable detection of associations of these variants with phenotypes beyond albuminuria.

\section{Clinical implications}

That the proteinuria in the Bedin et al. patient cohorts was albumin predominant yields important conclusions for practicing nephrologists: all albuminuria is not of glomerular origin, and isolated albuminuria may not be toxic to kidney function (4). Albuminuria has long been recognized as a hallmark of glomerular disease and indicative of a compromised filtration barrier that has lost size and charge selectivity. Although another genetic form of tubular proteinuria, Dent disease, results in kidney failure, interpretation is confounded by concomitant hypercalciuria and nephrocalcinosis. Here, Bedin et al. show that albuminuria can be associated with normal renal function in the context of CUBN loss of function (4). Identifying genetic causes of renal disease that are intractable to medical therapy is a growing area of clinical practice that is essential for avoiding unnecessary therapeutic or diagnostic interventions. For instance, seven of the patients in the Bedin et al. cohorts were treated with ACEI/ARB therapy without benefit, and others underwent renal biopsies that were nondiagnostic (4). The importance of genetic screening to help exclude unnecessary therapy is even more pointed in situations in which steroid treatment is being considered, such as in cases of focal sclerosing glomerulosclerosis (or in presumed cases of minimal change disease in children.

Ultimately, more longitudinal data will be required to definitively establish that long-term, isolated albumin-predominant proteinuria is truly benign. The diversity of proteinuria phenotypes that can occur in human disease and their subsequent, variable clinical consequences still remain to be fully appreciated. For instance, Bedin et al. draw a distinction between the albumin-predominant proteinuria resulting from $C U B N$ mutations and the proteinuria seen in LRP2 mutations that features a higher proportion of $\alpha 1$ - and $\beta 2$-microglobulins as well as a possible association with reduced eGFR $(4,25)$. This phenotypic distinction is striking, given that megalin and cubilin associate in the apical membrane of the proximal tubule and are coreceptors for a large repertoire of proteins, including albumin. That loss of function of one or the other of these coreceptors results in different patterns of proteinuria with differing clinical consequences is a remarkable fact that highlights how much there is yet to discover about proteinuria and its physiological consequences.

\section{Acknowledgments}

$\mathrm{AB}$ is supported by $\mathrm{NIH}$ grants T32DK108741 and TL1TR001875. JMB is supported by NIH grants 1U54DK104309, 2R01DK073462, and UG3 DK114926 and by a Columbia Precision Medicine Pilot Award. AGG is supported by NIH grants U01 DK100876 and R01 DK080099.

Address correspondence to: Ali G. Gharavi, Division of Nephrology, Columbia University Vagelos College of Physicians and Surgeons, 1150 St. Nicholas Avenue, Russ Berrie Pavilion 413, New York, New York 10032, USA. Phone: 212.851.4927; Email: ag2239@columbia.edu. 
1. Nielsen R, Christensen EI, Birn H. Megalin and cubilin in proximal tubule protein reabsorption: from experimental models to human disease. Kidney Int. 2016;89(1):58-67.

2. Kantarci S, et al. Mutations in LRP2, which encodes the multiligand receptor megalin, cause Donnai-Barrow and facio-oculoacoustico-renal syndromes. Nat Genet. 2007;39(8):957-959.

3. Aminoff $\mathrm{M}$, et al. Mutations in CUBN, encoding the intrinsic factor-vitamin B12 receptor, cubilin, cause hereditary megaloblastic anaemia 1. Nat Genet. 1999;21(3):309-313.

4. Bedin M, et al. Human C-terminal CUBN variants associate with chronic proteinuria and normal renal function. JClin Invest. 2020;130(1):335-344.

5. Kozyraki R, et al. The human intrinsic factorvitamin B12 receptor, cubilin: molecular characterization and chromosomal mapping of the gene to $10 p$ within the autosomal recessive megaloblastic anemia (MGA1) region. Blood. 1998;91(10):3593-3600

6. Andersen CB, Madsen M, Storm T, Moestrup SK, Andersen GR. Structural basis for receptor recognition of vitamin-B(12)-intrinsic factor complexes. Nature. 2010;464(7287):445-448.

7. Larsen C, Etzerodt A, Madsen M, Skjødt K, Moestrup SK, Andersen CBF. Structural assembly of the megadalton-sized receptor for intestinal vitamin $\mathrm{B}_{12}$ uptake and kidney protein reabsorption. Nat Commun. 2018;9(1):5204.

8. Storm T, et al. Detailed investigations of proximal tubular function in Imerslund-Gräsbeck syndrome. BMC Med Genet. 2013;14:111.
9. Cravedi P, Ruggenenti P, Remuzzi G. Proteinuria should be used as a surrogate in CKD. Nat Rev Nephrol. 2012;8(5):301-306.

10. Fried LF, Lewis J. Albuminuria is not an appropriate therapeutic target in patients with CKD: The Con View. Clin JAm Soc Nephrol. 2015;10(6):1089-1093.

11. Lambers Heerspink HJ, Gansevoort RT. Albuminuria is an appropriate therapeutic target in patients with CKD: The Pro View. Clin JAm Soc Nephrol. 2015;10(6):1079-1088.

12. Thompson A. Proteinuria as a surrogate end point - more data are needed. Nat Rev Nephrol. 2012;8(5):306-309.

13. Brenner BM, et al. Effects of losartan on renal and cardiovascular outcomes in patients with type 2 diabetes and nephropathy. N Engl J Med 2001;345(12):861-869.

14. Lewis EJ, Hunsicker LG, Bain RP, Rohde RD. The effect of angiotensin-convertingenzyme inhibition on diabetic nephropathy. The Collaborative Study Group. N Engl J Med. 1993;329(20):1456-1462.

15. Lewis EJ, et al. Renoprotective effect of the angiotensin-receptor antagonist irbesartan in patients with nephropathy due to type 2 diabetes. NEngl J Med. 2001;345(12):851-860.

16. Maschio G, et al. Effect of the angiotensinconverting-enzyme inhibitor benazepril on the progression of chronic renal insufficiency. The Angiotensin-Converting-Enzyme Inhibition in Progressive Renal Insufficiency Study Group. N Engl J Med.1996;334(15):939-945.

17. Hou FF, et al. Renoprotection of Optimal Antiproteinuric Doses (ROAD) Study: a randomized controlled study of benazepril and losartan in chronic renal insufficiency. J Am Soc Nephrol. 2007;18(6):1889-1898.

18. Bakris GL, et al. Renal outcomes with different fixed-dose combination therapies in patients with hypertension at high risk for cardiovascular events (ACCOMPLISH): a prespecified secondary analysis of a randomised controlled trial. Lancet. 2010;375(9721):1173-1181.

19. Fried LF, et al. Combined angiotensin inhibition for the treatment of diabetic nephropathy. N Engl J Med. 2013;369(20):1892-1903.

20. Mann JF, et al. Renal outcomes with telmisartan, ramipril, or both, in people at high vascular risk (the ONTARGET study): a multicentre, randomised, double-blind, controlled trial. Lancet. 2008;372(9638):547-553.

21. Parving HH, et al. Cardiorenal end points in a trial of aliskiren for type 2 diabetes. $N$ Engl J Med. 2012;367(23):2204-2213

22. Parving HH, Persson F, Lewis JB, Lewis EJ, Hollenberg NK, AVOID Study Investigators. Aliskiren combined with losartan in type 2 diabetes and nephropathy. $N$ Engl J Med . 2008;358(23):2433-2446.

23. Cravedi P, Remuzzi G. Pathophysiology of proteinuria its value as an outcome measure in chronic kidney disease. Br J Clin Pharmacol. 2013;76(4):516-523.

24. Böger CA, et al. CUBN is a gene locus for albuminuria. J Am Soc Nephrol. 2011;22(3):555-570.

25. Storm T, et al. Renal phenotypic investigations of megalin-deficient patients: novel insights into tubular proteinuria and albumin filtration. Nephrol Dial Transplant. 2013;28(3):585-591. 